\title{
Shrapnel Injury of Isolated Third Cranial Nerve
}

\author{
Murat Ulutaş ${ }^{1}$ Mehmet Seçer ${ }^{2}$ \\ ${ }^{1}$ Department of Neurosurgery, Sanko Hospital, Gaziantep, Turkey \\ 2 Department of Neurosurgery, Şehitkamil State Hospital, \\ Gaziantep, Turkey \\ J Neurol Surg Rep 2014;75:e217-e219.
}

\begin{abstract}
Address for correspondence Mehmet Seçer, MD, Department of Neurosurgery, Şehitkamil State Hospital, Osmangazi mah. 14 nolu Cadde 162/1 Şehitkamil Gaziantep, Turkey 27560

(e-mail: memetsecer@yahoo.com).
\end{abstract}

\begin{abstract}
Isolated third nerve palsy develops in numerous intracranial pathologies such as closed head trauma, tumor, and aneurysm. Isolated oculomotor nerve palsy caused by shrapnel injury is uncommon. After a penetrating intracranial shrapnel injury, our patient with oculomotor ophthalmoplegia underwent surgery. Microsurgery removed

Keywords

- third nerve

- shrapnel

- microsurgery the shrapnel that was applying pressure on the third nerve, resulting in contusion. A partial recovery associated with regeneration was observed at month 9. Extraocular muscle surgery should be planned if palsy does not resolve over a prolonged period of time.
\end{abstract}

\section{Introduction}

Cranial nerve injuries constitute a major portion of neurotraumas. ${ }^{1}$ These injuries can develop as a result of a direct traumatic impact, tissue reaction and an increase in intracranial pressure, or meningitis. ${ }^{2,3}$

Cranial nerve injuries are a major cause of morbidity because they require long-term follow-up, repeated surgeries, and reconstructive interventions. Along with major arteries and veins, cranial nerves support the brain that float in cerebrospinal fluid, and they also follow a route close to important vascular-neuronal structures. Cranial nerve injury may develop in the course of rapid acceleration/deceleration, shearing force, skull base injury, and penetrating cranial injury. ${ }^{1}$

\section{Case Report}

A 45-year-old man presented to our clinic on day 10 after his injury. A shrapnel-induced scalp laceration was present in the right temporal region, where the piece penetrated $3 \mathrm{~cm}$ above the zygoma. Immediately after the injury, he complained of right eyelid drop, diplopia, and blurred vision over short distances. In the neurologic examination, no deficits other than ophthalmoplegia (ptosis, dilated pupil, vertical limitation in eye movements and totally limited adduction, and the eye at the bottom-outer position at rest) were found. In the cranial computed tomography (CT) and CT angiography, a defect at the entry site to the right temporal bone, small bone fragments in the temporal parenchyma along the trace of the shrapnel and in the posterior-inferior portion of the posterior clinoid, and a metallic density localized in the prepontine cistern next to vascular structures were detected (-Figs. 1 and $\mathbf{2}$ ). A right pterional craniotomy and transsylvian approach was performed. Petechial contusion and thinning were noted in the third cranial nerve. Without compromising the integrity of the third cranial nerve, the $11-\mathrm{cm}$-long sharp-edged shrapnel, localized in the prepontine cistern next to the basilar artery and adhered to the nerve from the inferior with applied pressure was removed ( - Fig. 3 ) (video available at http:/l www.youtube.com/watch?v=rhuRBPl_2n4). No postoperative infection developed; a recovery by aberrant third nerve regeneration was observed at month 9 .

\section{Discussion}

The oculomotor nerve stems from the frontal surface of mesencephalon, advances forward in the subarachnoid space between the superior cerebellar artery and posterior cerebellar artery, and enters the lateral wall of the cavernous sinus by passing through the medial portion of the uncus. At the level of the superior orbital fissure, it divides into the ramus superior and ramus inferior branches. Along this course, fascicules are labeled as the subarachnoid segment, cavernous segment,
License terms

Stuttgart · New York

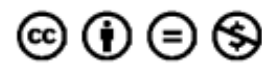

received

March 30, 2014

accepted

June 11,2014

published online

August 11, 2014
DOI http://dx.doi.org/

10.1055/s-0034-1387190. ISSN 2193-6358. 


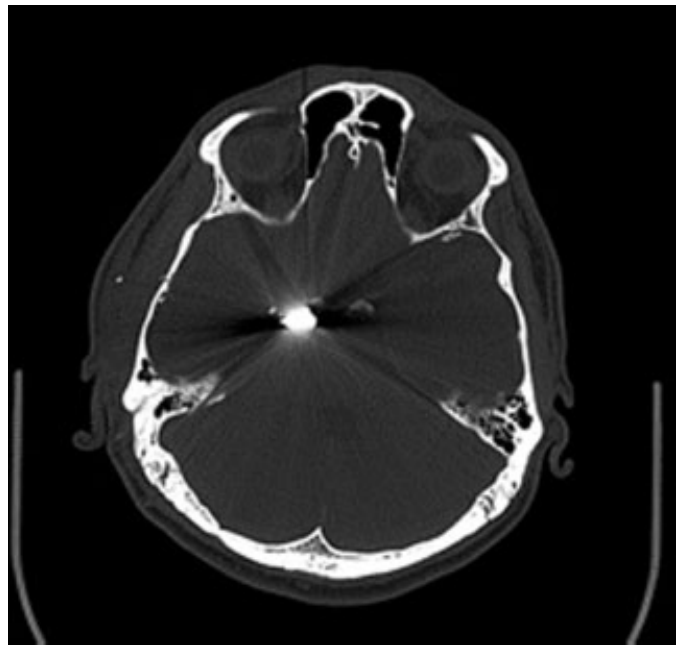

Fig. 1 Metallic artifact of the shrapnel in computed tomography.

orbital apex segment, and intraorbital segment, located in the neighborhood of the internal carotid artery (ICA), basilar artery and its branches, and the brainstem. ${ }^{2,4}$ (-Fig. 4).

Head trauma is responsible for 8 to $16 \%$ of oculomotor nerve palsies. ${ }^{2,5,6}$ Tectal hematomas, transtentorial herniation, and isolated oculomotor palsy induced by nerve avulsion or tension at the pontine-mesencephalic junction can develop. Multiple cranial nerve injuries involving the third nerve may occur in skull base fractures involving the cavernous sinus and in maxillofacial or superior orbital fissure injuries; isolated third nerve injury due to trauma is reported to be $21 \%^{2,7,8}$

Elston reported 20 cases with traumatic third nerve palsy, but no penetrating injury was present in their etiology. ${ }^{9}$ In 1400 case presentations, Keane reported traumatic oculomotor injuries in $26 \%$. Among those traumatic cases with gunshot injury, there were 28 cases with direct (injury form not specified) oculomotor nerve injury and 5 cases with oculo-

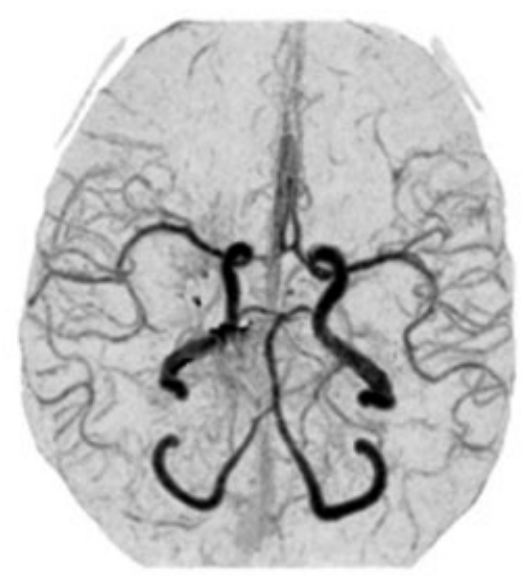

Fig. 2 Relation of the shrapnel to vascular structures in computed tomography angiography.

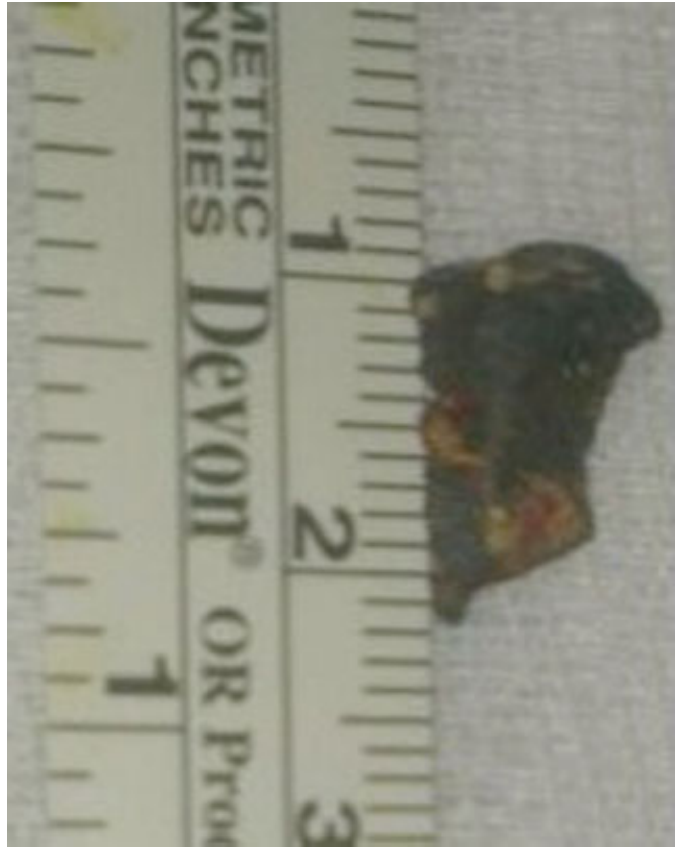

Fig. 3 The shrapnel.

motor nerve injuries secondary to herniation. ${ }^{2,8}$ In our case, pressure and/or blast impact caused by the shrapnel on the subarachnoid segment of the third nerve caused the palsy. It is very fortunate for the patient that the shrapnel did not damage vascular structures such as the ICA, basilar artery, and vital structures including the brain. The sharp-edged shrapnel was removed by microsurgery due to its risk of causing damage in the vascular-neural structures by pulsation and/or displacement. Pressure-dependent oculomotor palsy can be resolved by removing the pressure. ${ }^{10}$ However, in nonresolving oculomotor palsy, an extraocular muscle surgery may be required to restore binocular vision and the position of the eyes. $^{2}$ In third nerve palsy induced by trauma, an aberrant regeneration develops in $65 \%$ of cases. ${ }^{8}$ In our case, a partial recovery by an aberrant third nerve regeneration developed at month 9: horizontal gaze-eyelid synkinesis, pseudo-Von Graefe phenomenon (elevation of the lid on downward gaze), and limited elevation and depression of the eye with retraction of the globe with attempted vertical movements. The signs of nerve fiber misdirection may appear months to years after the third nerve injury occurs. Misdirection of

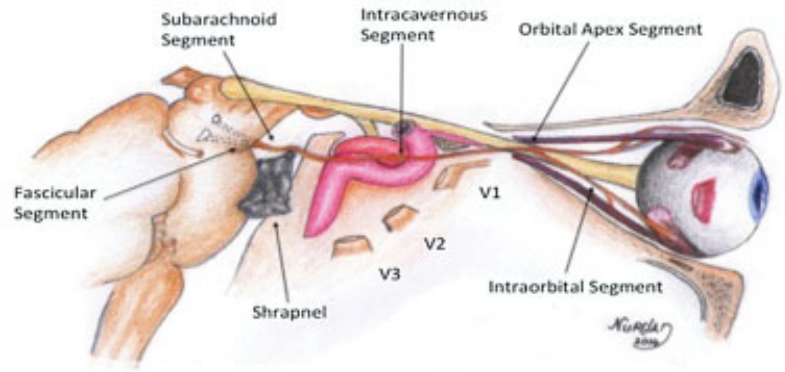

Fig. 4 Anatomic course of the third nerve and its relation to the shrapnel. 
regenerating third nerve fibers, ephaptic transmission, and central synaptic reorganization are the proposed mechanisms for this condition. ${ }^{11}$

\section{Conclusion}

Palsy of the oculomotor nerve localized next to important neural and vascular structures, resulting from the pressure of shrapnel, was observed in its subarachnoid segment. To prevent possible serious complications that may occur due to displacement of the sharp-edged shrapnel by pulsation and migration and to allow the recovery of the nerve by removing the pressure, the shrapnel should be removed by microsurgery. A detailed neuroradiologic evaluation should be performed before making the decision to operate. Surgery of the extraocular muscle should be planned in case palsy does not improve over time.

\section{Acknowledgments}

The authors would like to thank Nurdan Ulutaş for her assistance in preparing the manuscript.

Note

This case was presented as an electronic poster at the 28th Scientific Conference of the Turkish Neurosurgery Associ- ation. Written informed consent was obtained from the relative of the patient who participated in this study.

\section{References}

1 Patel P, Kalyanaraman S, Reginald J, et al. Post-traumatic cranial nerve injury. Indian J Neurotrauma 2005;2(1):27-32

2 Bhatoe HS. Trauma to the cranial nerves. Indian J Neurotrauma 2007;4(2):89-100

3 Keane JR, Baloh RW. Posttraumatic cranial neuropathies. Neurol Clin 1992;10(4):849-867

4 Foroozan R, Bhatti MT, Rhoton AL. Transsphenoidal diplopia. Surv Ophthalmol 2004;49(3):349-358

5 Rucker CW. The causes of paralysis of the third, fourth and sixth cranial nerves. Am J Ophthalmol 1966;61(5 Pt 2):1293-1298

6 Rush JA, Younge BR. Paralysis of cranial nerves III, IV, and VI. Cause and prognosis in 1,000 cases. Arch Ophthalmol 1981;99(1):76-79

7 McCormick WF. Pathology of closed head injury. In: Wilkins RH, Rengachary SS, eds. Neurosurgery. Vol 2. New York, NY: McGrawHill; 1996:2639-2666

8 Keane JR. Third nerve palsy: analysis of 1400 personally-examined inpatients. Can J Neurol Sci 2010;37(5):662-670

9 Elston JS. Traumatic third nerve palsy. Br J Ophthalmol 1984;68(8): 538-543

10 Park UC, Kim SJ, Hwang JM, Yu YS. Clinical features and natural history of acquired third, fourth, and sixth cranial nerve palsy. Eye (Lond) 2008;22(5):691-696

11 Slavin ML, Einberg KR. Abduction defect associated with aberrant regeneration of the oculomotor nerve after intracranial aneurysm. Am J Ophthalmol 1996;121(5):580-582 